\title{
Déterminants et actes de détermination en créole guadeloupéen
}

Juliette Facthum Sainton, Université des Antilles et de la Guyane

\section{Introduction}

Le sujet «déterminants et actes de détermination en créole guadeloupéen » a pour objectif la mise en perspective des déterminants et du système de détermination du créole guadeloupéen. Il répond à un souci, celui de contribuer à pourvoir le créole guadeloupéen, créole à base lexicale française de l'archipel de Guadeloupe dans la mer Caraïbe (Basse-Terre, Grande-Terre, Marie-Galante, Terre-de-Haut et Terre-deBas, et la Désirade), d'éléments pour une analyse grammaticale et linguistique. Il n'existe aucun ouvrage qui fasse référence scolaire, universitaire ou même populaire, en matière de grammaire du créole guadeloupéen (CG).

Nous avons choisi dans cette première étape d'exploration de la grammaire du guadeloupéen de travailler sur la détermination parce que c'est une thématique qui permet de prendre du recul non pas seulement par rapport aux déterminants, mais encore par rapport au nom, et plus particulièrement en liaison avec les rapports syntagmatiques entre le déterminant et le nom, et les rapports paradigmatiques particuliers entre déterminants en créole, la distribution du déterminant n'étant pas la même, selon que le déterminant a une valeur définie ou une valeur indéfinie. Nous y examinerons la question du nombre et du genre. Dans une brève perspective diachronique, les ré-analyses du français (grammaticalisations), déconstructions (francisations) et réinterprétations (nouvelles créolisations) seront interrogées. La différence entre nature et fonction est l'indispensable complément à une analyse grammaticale de la détermination en créole.

Toute entreprise descriptive des créoles se situe au carrefour d'une kyrielle de préoccupations :

- l'urgence pour les sociétés créolophones, à un stade de développement où la mondialisation occupe une place importante dans les microsociétés, de pouvoir exprimer leur pensée le plus justement et le plus précisément possible, dans la « langue de l'environnement » qu'est le créole.

- l'entreprise de conciliation, dans une même grammaire, pour une même partie du discours et concernant la même nature grammaticale, de traits, fonctions et 
structures appartenant à des niveaux de créole différents : un basilecte plutôt ancien représentant aujourd'hui à l'école le bon usage, idéal à atteindre, et un acrolecte représentant le niveau récent, de francisation le plus achevé du créole. Ces deux niveaux clichés aux antipodes l'un de l'autre laissent voir d'autres niveaux intermédiaires d'un continuum alimenté par un processus constant de ré-analyses, de déconstructions, de réinterprétations. Cette dynamique est assez spécifique aux créolophonies: les langues créoles, du moins celles nées de la colonisation du Nouveau Monde et de l'Océan indien par l'Europe, évoluent dans un type de contacts de langues sans discontinuité temporelle avec les langues européennes qui ont présidé à leur naissance.

- l'intégration de la question diachronique créole, incontournable pour la formation et le discernement didactique de l'enseignant de français et de créole en milieu créolophone. Les deux langues sont apparentées et intimement liées au quotidien, et pourtant elles constituent des codes linguistiques différents.

\section{Déterminants et détermination en CG}

\section{Définition du terme « déterminant »}

Nous proposons ici de partir de l'aphorisme de la grammaire traditionnelle définissant le déterminant comme une catégorie grammaticale qui actualise le nom. D'ailleurs, on s'en réfère souvent par une lapalissade, «le déterminant du nom ». On acceptera la critique qu'une telle définition est sommaire. Mais dans l'état d'amorce de la présente étude, elle suffit à alimenter nos propos. On s'accordera sur ce qui semble également être une évidence universelle : le déterminant ne peut être utilisé seul en tant que constituant; sa présence ne peut être justifiée que par l'emploi d'un nom $(\mathrm{N})$. On en conclura donc de l'axiome que le déterminant fait partie du syntagme nominal (SN). La chaîne parlée On timoun est un syntagme nominal dans la phrase On timoun ka kouri an lakou-la (un enfant gambade dans la cour).

Identifier le déterminant et le syntagme nominal au sein de quelle unité de parole ?

Ce n'est pas tout d'avoir identifié un SN. Tout syntagme considéré isolément, hors contexte, constitue difficilement une chaîne parlée suffisante pour la description. Nous proposons ici une unité de parole plus large au sein de la chaîne, à savoir la phrase. Nous préférons cet outil conceptuel à celui autre que représente l'énoncé. La 
phrase est une référence légitimée par les néophytes. En particulier, la phrase simple est le repère permettant l'identification des constituants minimums d'un acte de parole, à savoir, universellement, le SN (Syntagme nominal) en fonction sujet grammatical, et le SP (Syntagme prédicatif): On timoun ka pasé, est une phrase simple. Cette dernière est constituée d'un $\mathrm{SN}$, on timoun, en fonction sujet, et d'un syntagme prédicatif (SP), ka pasé. Il n'a cependant pas encore été question du déterminant.

\section{L'attestation de la catégorie grammaticale des déterminants en créole}

La troisième étape consistera à identifier et à attester la présence d'éventuels constituants qui soient des déterminants au sein d'un SN en créole guadeloupéen. Dans la phrase, on timoun ka pasé, il s'agit du déterminant indéfini on, dont la variante libre est an. Il est placé devant le nom timoun qu'il actualise : on timoun/an timoun. En grammaire traditionnelle, le déterminant on exprime que le locuteur, auteur de cette phrase, n'a pas d'informations sur l'identité de l'enfant en question. Dans une perspective plus linguistique, le déterminant indéfini extrait au hasard un objet de la catégorie décrite par le SN (Manuélian et Fattier). Ces deux auteurs à propos du créole haïtien ainsi que Lapaire et Rotgé à propos de l'anglais (95-100) préconisent que le déterminant indéfini peut être paraphrasé par «n'importe quel $\mathrm{N}$ parmi tous les $\mathrm{N} \gg$.

Le déterminant indéfini étant attesté, c'est la catégorie des déterminants ainsi que le Constituant Déterminant qui est attesté en créole guadeloupéen.

En grammaire linguistique, la notion d'indéfini implique non pas l'absence d'identification précise du référent :

Un référent indéfini peut être spécifique : On timoun sòti pasé la (un enfant vient de passer). Le SN on timoun se réfère spécifiquement à l'enfant qui vient de passer et à aucun autre enfant.

Un référent indéfini peut être non-spécifique : an ka chèché on timoun solid an péyi-lasa (je cherche un enfant courageux dans ce pays). Dans ce cas, le SN on timoun se réfère à un enfant dont l'identité est non-spécifique.

Il est admis, selon les descriptions les plus récentes, que le non-spécifique va jusqu'à une interprétation générique (Manuélian et Fattier 4). En créole guadeloupéen, cela donnera la phrase suivante : on nonm pa dwèt fè kankan « un homme (les êtres de sexe masculin) ne devrait pas faire de cancans ». 
Rappelons qu'il a été attesté ci-dessus que le déterminant on/an du créole guadeloupéen correspond à la distribution des déterminants antéposés au N. On écrira alors : $\mathrm{SN}=$ Déterminant indéfini+N.

Outre le déterminant on, un paradigme des déterminants antéposés est-il attesté en créole?

En faisant commuter on avec un autre constituant actualisant le $\mathrm{N}$, il sera possible d'attester la présence d'un paradigme des déterminants antéposés au nom :

On timoun ka pasé (un enfant passe)

Dotwa timoun ka pasé (quelques enfants passent)

Onlo timoun ka pasé (beaucoup d'enfants passent)

Onpil timoun ka pasé (beaucoup d'enfants passent)

Les exemples ci-dessus, mis à l'épreuve de la commutation, peuvent laisser perplexe, tout en confirmant l'existence d'un paradigme des déterminants antéposés au nom: en effet, on peut se demander si l'identification de tels déterminants antéposés ne sont pas du «construit » de linguiste ou si ce n'est pas simplement on+N (lo/pil). Ces catégories grammaticales sont vraisemblablement le résultat étymologique de la prothèse de l'article indéfini un et des substantifs lot et pile dont le glissement de sens a pu donner, déjà en français populaire, le sens de beaucoup de.

Quelles structures pour le SN lorsque le déterminant est antéposé au nom?

Le déterminant on est antéposé au nom. Le syntagme nominal est donc constitué comme suit :

Déterminant-Noyau nominal : on- timoun

Ce déterminant indéfini est-il inséparable du nom ou noyau nominal qu'il actualise comme est suggéré dans l'exemple on timoun ?

En prenant un autre exemple dans une nouvelle phrase, on bèl timoun ka pasé (un bel enfant passe), on peut affirmer que le déterminant indéfini et le nom ne sont pas inséparables ; il peut y avoir un constituant adjectif entre les deux. Cet adjectif bèl, toujours antéposé au nom, fait partie d'une liste fermée d'adjectifs antéposés au N. Cette liste est composée exclusivement de bèl, gran, jenn, vyé, piti, ti, gwo, bidim (beau, bel(le), grand(e), jeune, vieux/vieil/vieille, petit(e), minuscule, cher/chère, gros(se), imposant(e) ). Dans ce cas, le SN, lorsque détaillé, est constitué comme suit :

Déterminant-Adjectif-Noyau nominal : on-bèl-timoun 
Mais, s'il s'agit d'un autre adjectif à usage postposé au nom, s'inscrivant davantage dans un commentaire où intervient un parti-pris, le déterminant et le nom se situent à nouveau dans la continuité l'un de l'autre : on timoun gyòk sòti pasé la (un enfant gaillard vient de passer). Dans ce cas, l'ordre des constituants est le suivant :

«Déterminant-Nom -Adjectif » : on-timoun-gyòk

\section{L'attestation d'un déterminant indéfini dans une langue, implique-t-elle un déterminant défini ?}

En créole comme en français, et dans les langues romanes ou issues en partie des langues romanes, il existe un déterminant à valeur indéfinie. Il existe également un déterminant à valeur définie. Soit la phrase suivante : Timoun-la sòti wou pasé la (l'enfant vient de passer à nouveau).

A partir du moment où l'on compare les deux phrases, on timoun sòti pasé et timoun-la sòti woupasé la, le seul indicateur, wou-, met en contraste le groupe de constituants, on timoun et timoun-la. De ce fait, «-la» dans timoun-la apparaît comme un déterminant, indiquant formellement l'identité de l'enfant, celui qui est déjà connu du locuteur, parce que déjà passé une première fois dans les parages. Le constituant -la ne peut donc être identifié que comme un déterminant défini.

Le déterminant défini, -la du créole, est postposé au nom. En ce sens, la distribution de ce déterminant défini du créole atteste une divergence d'avec celle du français. En français, le déterminant défini dont les formes sont $l e, l a, l$ ', est toujours antéposé au nom.

En grammaire linguistique, on considère que $-l a+\mathrm{N}$ peut s'interpréter comme une anaphore textuelle, c'est-à-dire que N s'interprète à partir d'un segment déjà énoncé, dans le contexte à gauche : On timoun té ka vini asi mwen. Lè timoun-la rivé pwé mwen, ka an ka vwè : sé Woni gason a'w « un enfant se dirigeait vers moi » / « Lorsqu'il arriva près de moi, je réalisai que c'était Rony ton fils ». C'est le segment d'énoncé On timoun té ka vini asi mwen qui explique l'usage anaphorique du SN timoun-la.

L'anaphore peut être culturelle : si ou fè pipi asi’w, métrès-la péké kontan «si tu fais pipi sur toi, la maîtresse ne sera pas contente » dit une mère à son enfant de trois ans qui n'a jamais été à l'école. Selon les études faites, l'usage de -la, dans le SN métrès-la, jette un pont de connaissance culturelle entre la mère et son enfant: 
dans toute classe, il y a un maître et en Guadeloupe le plus souvent, une maîtresse d'école.

L'anaphore peut être situationnelle : pwan on sèvyèt an lanmwa-la ban mwen, souplé (prends-moi une serviette dans l'armoire, s'il-te-plaît), dit un malade à une vieille amie venue lui rendre visite. Le locuteur part du principe que son amie a déjà repéré l'armoire.

On remarquera qu'en créole, contrairement au français, le générique ne se marque pas par l'emploi de l'article défini singulier ou pluriel. Il se marque soit par l'usage de on (Rappel : on nonm pa dwèt fè kankan; un homme ne doit pas faire de cancans), soit par l'usage d'un $\mathrm{N}$ nu, non déterminé (nonm pa dwètèt fè kankan; les hommes ne doivent pas faire de cancans).

Enfin, le guadeloupéen est le seul créole à base lexicale française de la Caraïbe qui n'a pas de variantes contextuelles du déterminant défini -la. Hormis quelques rares idiolectes, la forme incontestée est -la. En Martinique par exemple, le contexte phonétique donne lieu à des variantes telles an, wan, $a$, wa, la : jaden-an (le jardin), pon-wan (le pont)- madjoumbé-a (la fourchette), labou-wa (la boue), tab-la (la table). A Sainte-Lucie, kann-nan (la canne).

Mise en relation syntaxique directe ou indirecte entre le nom et le déterminant défini ?

Soit l'exemple suivant: Bèl timoun-la sòti pasé la ankò. Le GN est bèl timoun-la. Il est composé des constituants suivants : Adjectif- Nom-Déterminant.

Mais, si l'adjectif n'est pas compatible avec l'antéposition, ce qui est le cas pour la majorité des adjectifs du créole, timoun gyòk la sòti pasé la, alors, le SN admet l'ordre suivant des constituants : Nom-Adjectif-Déterminant.

En conclusion des paragraphes ci-dessus, on note que l'hypothèse d'un déterminant indéfini et d'un déterminant défini est confirmée. L'existence d'une catégorie grammaticale, celle des déterminants du créole, est donc fondée.

Fondé sur le déterminant -la, un paradigme des déterminants postposés peut-il être attesté ?

L'expression de la possession, qui souvent dans les langues mais pas toujours, se rapporte à un acte de détermination, est postposée en créole : chapo an mwen (mon chapeau/mes chapeaux), chapo $a^{\prime} w$ (ton/tes chapeau(x)), chapo $a^{\prime} y$ (son/ses 
chapeau(x)), chapo an nou (nos chapeaux), chapo a zòt (votre/vos chapeau(x)), chapo a yo (leurs chapeaux).

Il est connu que les créoles marquent la possession, non pas à l'aide d'un déterminant possessif, mais à l'aide d'un pronom personnel placé après le nom. En créole guadeloupéen, le constituant $a$ devant les pronoms en contexte oral (chapo a yo) et an en contexte nasal (chapo an mwen) est un constituant de mise en relation (de dépendance) entre le référent possédé à gauche et le possesseur à droite. Contrairement à l'attestation d'une catégorie grammaticale de déterminants possessifs en français, en créole il n'existe pas de déterminant possessif. Il existe un acte de détermination actualisant la relation entre l'unité lexicale indiquant la chose possédée et le possesseur qui est un pronom personnel.

Le statut de déterminant du constituant possessif du créole est mis en cause, car ce constituant est compatible, avec le déterminant -la : chapo an mwen-la, chapo a'w-la, chapo a'y-la, chapo an nou-la, chapo a zòt-la, chapo a yo-la.

A notre avis, la détermination possessive, dans les langues, donne au référent actualisé une valeur « définie ».

En créole guadeloupéen, il existe un constituant « déterminant» composé de « déterminant défini -la + valeur démonstrative $s a$ (définie). Il existe donc un acte de détermination «sur-défini ». En effet, le constituant «déterminant » permet à la fois de dire l'appartenance du chapeau an mwen, $a$ 'w, ...) et en plus qu'il en était déjà question antérieurement (-la).

Il convient ici de lever une ambiguïté sur l'acte de détermination possessive en créole guadeloupéen et sur lequel les linguistes butent: il y a une différence sémantique entre chapo an mwen et chapo an mwen la. Dans le premier GN, il s'agit d'un chapeau que l'allocutaire n'a pas nécessairement vu auparavant. Dans le deuxième GN, le déterminant -la indique, qu'il s'agit d'un chapeau dont il a déjà été question entre le locuteur et son allocutaire.

Le deuxième acte de détermination postposé est celui de la monstration, c'està-dire la valeur démonstrative : an enmé chapo-lasa. En créole guadeloupéen, le constituant $s a$, à valeur démonstrative, est postposé au nom. Cependant, ce constituant n'est pas un déterminant, car il n'est jamais attesté seul, sans le déterminant -la. Il permet de faire un acte de détermination démonstrative, après $l a$.

Chapo-la : le chapeau-chapo-lasa : ce chapeau 
Conclusion : il existe bien un paradigme du déterminant postposé en créole. Il est fondé par -la compatible avec le pronom personnel qui lui est antéposé et le constituant non autonome à valeur démonstrative qui lui est postposée. L'ensemble de ces constituants sont compatibles les uns avec les autres dans un acte de détermination postposé : timoun a'w-lasa anmègdan mot-à-mot : enfant de toi le cet est espiègle.

\section{Quels sont les règles et principes ?}

Il existe une catégorie grammaticale du déterminant reposant sur l'attestation de deux déterminants centraux sémantiquement, le déterminant indéfini on et le déterminant défini-la. Il existe par ailleurs, un paradigme de la détermination antéposée (on, onlo, onpil, détwa, titak, tigout, tibwen, on, dé, twa...) et un paradigme de la détermination postposée (-la, an mwen... ; an mwen-la ; lasa, an mwen lasa).

Il y a-t-il un lien entre valeurs sémantique des déterminants et distribution?

Il existe un lien entre la valeur indéfinie du déterminant et sa distribution antéposée : tous les quantifieurs, qui n'ont qu'une valeur indéfinie sont antéposés au nom.

Il existe bien un lien entre la valeur définie du déterminant et sa distribution postposée : tous les constituants qui participent à l'acte de détermination postposée ont une valeur définie.

Ainsi, il y a un lien entre valeur indéfinie et distribution antéposée et valeur définie et distribution postposée. Ce trait est valable pour tous les créoles à base lexicale française des Petites Antilles. La distribution n'est pas la même en guyanais. La description de l'haïtien présente également quelques divergences avec les créoles des Petites Antilles en matière de déterminants et de détermination (voir sur ce point Damoiseau, dans ce dossier).

\section{Comment concilier dans une même catégorie grammaticale, dans une même grammaire, deux constituants dont la distribution respective est différente ?}

\section{Une problématique importante}

Se pose une problématique importante : celle de savoir comment concilier dans une grammaire, deux constituants qui appartiennent à la même catégorie grammaticale mais qui n'auraient pas la même distribution ? La commutation de deux éléments, au plan syntagmatique, comme au plan paradigmatique ne porte pas de 
réponse à la question posée. En effet, le déterminant -la ne peut jamais commuter avec on.

C'est au plan de la grammaire générative de Noam Chomsky que singulièrement une réponse est nettement amorcée : la grammaire générative part du principe que chaque syntagme est une unité centrale dans laquelle il y a une unité de rang supérieur et une unité de rang inférieur. Dans une phrase simple, le SN et le SP constituent deux unités centrales. Chaque unité centrale est elle-même constituée d'un noyau central ou unité de niveau supérieur et d'une unité de niveau inférieur. Le $\mathrm{N}$ s'impose comme unité de niveau supérieur : sans le nom, la présence du déterminant ne se justifie pas. En effet, on ne peut dire en créole : * On ka pasé. « un/une vient ». Pour que cet énoncé devienne compréhensible, il faudrait qu'il y ait un nom comme dans « l'énoncé » On timoun ka pasé « Un enfant passe ».

Le déterminant apparaît alors comme une unité de niveau inférieur, car sa présence ne se justifie pas sans un nom. On peut dire timoun ka pasé sans le déterminant antéposé ou postposé. Ainsi, on peut partir du raisonnement selon lequel étant donné que deux unités de niveau inférieur on et la actualisent également le noyau nominal, on peut concevoir que même sans partager la même distribution, ces derniers appartiennent au même répertoire de catégorie grammaticale, celui des déterminants.

\section{Existe-t-il des distributions autres que l'antéposition et la postposition ?}

Il importe de comprendre que les deux constituants fondateurs de la valeur indéfinie et de la valeur définie, respectivement on et -la, ne sont pas compatibles entre eux. L'usage de on dans une phrase exclut celui de -la. Il existe cependant une distribution de l'acte de détermination qui combine des constituants antéposés du groupe «Déterminant » à d'autres constituants postposés de ce même groupe. La phrase suivante en est une illustration : Lòt timoun-la pa vini (l'autre enfant n'est pas venu). Dans cette phrase, le groupe « Déterminant» est « discontinu ». Il est composé du qualifieur indéfini lòt et du déterminant défini -la.

Le même type de distribution atteste en position postposée, la combinaison linéaire déjà étudiée ci-dessus, du déterminant, -la, à la marque du possessif an mwen: lòt timoun an mwen-la pa vini (mon autre enfant, celui dont on avait parlé auparavant, n'est pas venu). 
Nous ne pouvons ici continuer l'étude de la distribution sans entrer dans des considérations sur la catégorie du nombre. Même si la catégorie du genre n'a aucune conséquence sur la relation syntagmatique entre nom et déterminant, il convient d'explorer également une telle catégorie. Genre et nombre permettent d'en dire plus sur la morphologie du déterminant dans les langues du monde.

Problématique de la catégorie du genre des déterminants et du nom en guadeloupéen : ré-analyse, déconstruction, réinterprétation

La catégorie du genre n'existe pas. Ni le déterminant ni le nom n'est soumis à une telle catégorie. Il existe en diachronie une liste fermée de mots du créole qui par ré-analyse du français, commencent par un préfixe non autonome la- qui pour intégrer le créole ont fait la prothèse de l'article défini la du français au $\mathrm{N}$ féminin. Il s'agit de mots tels lalin, lapli, lawouzé, lari, lavwari, lanmè, lanméri. Dans ce cas, le mot créole ne peut être considéré comme féminin. Le préfixe non autonome avec lequel fonctionne le mot fait partie intégrante de sa morphologie et n'a pas de valeur déterminative. En bref, ce préfixe n'est pas un article défini.

Dans l'état synchronique actuel, le déterminant défini s'exprime en position finale de ces mêmes mots : lalin-la plenn. Lapli-la pa tonbé. Lari-la gran. Lanmè-la mové dans les Amériques.

Il semble y avoir eu, en particulier en ce moment, une déconstruction populaire, à la lumière des apprentissages en français : lapli paka tonbé koté an mwen (la pluie ne tombe pas près de chez moi). Si loraj woulé, lanmè ké monté (Si le tonnerre gronde, la mer sera grosse). Ici, la tendance pourrait être le découpage grammatical suivant : Si l'oraj woulé, lan mè ké monté. De même, la pli paka tonbé koté an mwen. Ces types de réinterprétations, erronées, sont cependant rendues possibles par l'absence de la détermination postposée : lapli-la au lieu de lapli.

La déconstruction va plus loin ici. Elle conduit à des réinterprétations grammaticales et sémantiques : comme il existe en créole une valeur générique du nom, non déterminé, fréquemment attestée, par exemple dans l'adage, timoun pa ti chodyè, il en résulte un emploi du générique, y compris avec les mots commençant par le préfixe non autonome la-. Ces usages où lapli-la côtoie lapli semblent se limiter exclusivement aux îles de Guadeloupe dans les Petites Antilles et méritent que la réinterprétation soit posément analysée comme suit : 
- Lapli paka tonbé koté an mwen (il ne pleut pas près de chez moi). Les mots se référant aux éléments atmosphériques ont un emploi indéterminé lorsqu'ils sont utilisés en valeur abstraite.

- Lapli-la ban nou on favè (la pluie nous a donné une faveur). Ces mêmes mots peuvent avoir un emploi déterminé, lorsque utilisés en valeur concrète, matérialisée : il s'agit de ce mauvais temps-ci/là, et pas de celui qui a sévi la semaine précédente. Ceci nous ramènera en aval à explorer l'étymologie française de l'article défini postposé créole, -la.

\section{Le déterminant, le nom et la catégorie du nombre}

La catégorie du nombre du déterminant et du nom est attestée dans les corpus du créole guadeloupéen. Sa manifestation est divergente de celle du français.

Le déterminant indéfini, on, n'a pas de forme correspondante au pluriel. Il se manifeste par une absence de détermination : ni on tiboug an lakou lékòl-la/ Ni tiboug an lakou lékòl-la (il y a un garçon dans la cour de récréation/ il y a des garçons...). Le nom n'a pas non plus de forme du singulier ou de forme du pluriel.

Le déterminant défini, -la, garde la même forme dans l'expression de la singularité et de la pluralité. Il en est de même du nom. La pluralité est marquée par un constituant antéposé au nom, sé, le «pluralisateur ${ }^{1}$ »: Tiboug-la an lakou lékòl-la toujou/Sé tiboug-la an lakou lékòl-la toujou.

La pluralisation du déterminant défini, $-l a$, est l'une des grandes divergences entre les créoles des Petites Antilles qui utilisent tous le pluralisateur sé, et d'une part, le haïtien qui atteste la transformation de -la en yo, et d'autre part le guyanais qui atteste $y a$

Sé fanm-la (Guad) - Sé fanm-lan (Mar) - Sé fanm-nan (Ste Lucie)- fanm yo (H)- fanm ya (Guy) «les femmes » dans un contexte anaphorique.

\section{Dénombrables vs indénombrables}

Le déterminant indéfini, on, donne au nom une valeur dénombrable. Il en est de même du déterminant défini -la. Mais, il existe une opposition dénombrable/indénombrable au sein du système de détermination :

Le déterminant onlo est compatible avec les noms, dénombrables et indénombrables : Ni onlo luil an salad-la- Ni onlo moun ki vlé salad.

Les déterminants dotwa et tibwen s'opposent et se complètent: le premier n'est compatible qu'avec les noms dénombrables et le deuxième seulement avec les 
noms indénombrables: Ni dotwa mango (il y a quelques mangues), men ni tibwen diven (mais il y a un peu de vin).

\section{Le point de saturation du constituant « Déterminant » défini}

Timoun-la ka dòmi

Timoun a'w-la ka dòmi

Lòt timoun-la ka dòmi

Lòt timoun a'w-la ka dòmi

Lòt timoun a'w-la-sa ka dòmi.

Au singulier, le point de saturation du constituant « Déterminant» défini, est : lòt...a'w-la-sa. Ce constituant est formé de quatre sous-constituants, discontinus et se présentant sous la compatibilité « antéposé...postposé » et composé comme tel :

Quantifieur/ qualifieur....possession- déterminant défini- monstration.

\section{En conclusion}

\section{Epistémologie, diachronie et grammaticalisation}

Si l'on se base sur les propos de J.R. Lapaire et W. Rotgé, le processus d'émergence du déterminant défini d'une langue que nous appellerons ici langue $B$, tire souvent son origine du démonstratif et/ou de l'adverbe d'espace-lieu et de temps d'un état antérieur de langue $A$. C'est ainsi qu'ils rattachent the de l'anglais contemporain, disons $B$, aux adverbes d'espace-lieu-temps there, then, correspondant à un état antérieur de langue anglo-saxonne, $A$.

Le déterminant défini postposé du créole, -la, porte en lui la trace d'un démonstratif du français qui peut également être l'adverbe là du français. Contrairement aux idées reçues, la position postposée du déterminant du créole n'est pas due à l'inversion du GN féminin du français, sinon pourquoi les noms masculins en français et passés dans le créole prendraient-ils également le même déterminant-la. C'est que «là » adverbe, avant la mutation qui aboutit au créole était déjà postposé au nom en français. Certains usages de -la en créole gardent la trace de cette valeur démonstrative et adverbiale.

Les exemples qui suivent tendent à retracer l'origine adverbiale d'ancrage spatiale locative de la créole, puisqu'il entre dans la composition d'actes d'adverbialité de lieu que le locuteur peut identifier : dèwò-la, andidan-la, douvan-la, dèyè-la, kotésit-la ${ }^{2}$. L'enseignement du créole en Guadeloupe semble avoir réalisé la prothèse graphique, et grammaticale : dèwòla, andidanla, douvanla, dèyèla, kotésitla. 
L'une des difficultés de la prothèse graphique est la difficulté de découpage d'un mot à quatre syllabes tel andidanla et la difficulté à segmenter les graphèmes contigus -tldans kotésitla.

Ces autres exemples tendent à suggérer la trace d'une valeur «monstrative » ancienne de là, dans la formation des adverbes de temps suivants du créole : bomatenla, aprémidi-la, oswè-la, toutalè-la. Les traductions anglaises et françaises this morning/ce matin, this afternoon/cet après-midi, this evening/ce soir, avec this/ce appuient la thèse de la trace d'un déterminant démonstratif à l'origine de -la.

\section{Répertoire de l'acte de détermination du créole guadeloupéen}

L'article indéfini antéposé on indique 1) que l'identité du référent est inconnu ou 2) qu'il y a extraction d'un référent, alors que l'article défini postposé -la indique : 1) qu'un référent est situationnel, 1) qu'il est référentiel, 3) que son identité est connue du locuteur et de son interlocuteur.

L'acte de non détermination indique, dans l'ensemble, que : 1) l'identité des référents au pluriel est inconnue ; 2) le nom est générique ; 3) il s'agit d'une valeur référentielle.

A cela, ajoutons que les constituants démonstratifs la-sa localisent dans l'espace et dans le temps, dans une dépendance l'un de l'autre, les possessifs renseignent sur le «possesseur» et fonctionnent selon la personne (an mwen, a'w, $a^{\prime} y$, an nou, a zòt, $a$ yo) en étant précédés d'un constituant de mise en relation $a$ (dont l'allomorphe est an en contiguïté d'un contexte nasal postérieur). Là où les déterminants cardinaux renseignent sur le nombre, les quantifieurs indéfinis renseignent sur la quantité indéterminée (onlo, onpil, tout, chak, plizyé, dotwa, tibwen, tigout, titak, pa-on, ayen, ahak). Enfin, le déterminant interrogatif indique que la question porte sur le nom (le constituant exclamatif, lui, actualise le nom), tandis que le déterminant situationnel $l i$ indique qu'il s'agit d'un titre important et que l'identité de la personne est unique, ce qui diffère de la antéposé (la mayé), lequel détermine un référent universel (la mariée).

\section{Références bibliographiques}

Bernabé, J. «Le constituant Déterminant». Fondal-natal. Grammaire basilectale approchée des créoles guadeloupéen et martiniquais, volume 2. Paris: L'Harmattan, 1983. 631-764. 
---. «Les modalités nominales ». Précis de syntaxe créole Ibis Rouge, 2003. 107-129.

Damoiseau, R.: Eléments de grammaire comparée Français-Créole, Ibis Rouge, 1999.

Facthum Sainton, J. Adaptation du français aux situations de créolophonie. Guide du maître : la Guadeloupe, 2010.

Manuélian, H. et D. Fattier. L'utilisation des déterminants en créole haïtien : étude de quelques chaînes de référence. http://hal.archives-ouvertes.fr/docs, 2011.

Lapaire, J-R. et W. Wotgé. Linguistique et grammaire de l'anglais. Toulouse : PU du Mirail, 1991.

\footnotetext{
Notes

${ }^{1}$ Dans l'ouvrage Précis de syntaxe créole (1983), Jean Bernabé définit sé dans sé kakat-bwa ka pété zorey-mwen «les grillons me brisent le tympan », par le terme «pluralisateur», pp. 108. A propos du pluriel de la valeur démonstrative du déterminant, il utilise ce même terme (113) : Sé jou tala, man pa djè ni lajan « Ces jours-ci je n'ai guère d'argent.

${ }^{2}$ Contrairement à certains usages en cours, nous avons adopté ci-dessus une graphie en deux mots mis en relation syntaxique par un trait d'union, par souci de déchiffrement syllabique rapide par le lecteur non expert.
} 